\title{
Site-Resolved Contributions to the Magnetic-Anisotropy Energy and Complex Spin Structure of $\mathrm{Fe} / \mathrm{MgO}$ Sandwiches
}

\author{
Ramón Cuadrado, ${ }^{1,2,3,{ }^{*}}$ László Oroszlány, ${ }^{4}$ András Deák, ${ }^{5}$ Thomas A. Ostler, ${ }^{6,7}$ Andrea Meo, ${ }^{1}$ Roman V. Chepulskii, ${ }^{8}$ \\ Dmytro Apalkov, ${ }^{8}$ Richard F. L. Evans, ${ }^{1}$ László Szunyogh, ${ }^{5,9}$ and Roy W. Chantrell ${ }^{1}$ \\ ${ }^{1}$ Department of Physics, University of York, York YO10 5DD, United Kingdom \\ ${ }^{2}$ Catalan Institute of Nanoscience and Nanotechnology (ICN2), CSIC and BIST, \\ Campus UAB, Bellaterra, 08193 Barcelona, Spain \\ ${ }^{3}$ Universitat Autonoma de Barcelona, 08193 Bellaterra (Cerdanyola del Valles), Spain \\ ${ }^{4}$ Department of Physics of Complex Systems, Eötvös University, \\ Pázmány Péter sétány 1/A, H-1117 Budapest, Hungary \\ ${ }^{5}$ Department of Theoretical Physics, Budapest University of Technology and Economics, \\ Budafoki út 8, H-1111 Budapest, Hungary \\ ${ }^{6}$ Faculty of Arts, Computing, Engineering and Sciences, Sheffield Hallam University, \\ Howard Street, Sheffield, S1 1WB, United Kingdom \\ ${ }^{7}$ Department of Physics, Université de Liège, B-4000 Liège, Belgium \\ ${ }^{8}$ Samsung Electronics, Semiconductor R and D Center (Grandis), San Jose, California 95134, USA \\ ${ }^{9}$ MTA-BME Condensed Matter Research Group, Budapest University of Technology and Economics, \\ Budafoki út 8, H-1111 Budapest, Hungary
}

(Received 4 December 2017; revised manuscript received 9 April 2018; published 30 May 2018)

$\mathrm{Fe} / \mathrm{MgO}$-based magnetic tunnel junctions are among the most promising candidates for spintronic devices due to their high thermal stability and high tunneling magnetoresistance. Despite its apparent simplicity, the nature of the interactions between the $\mathrm{Fe}$ and $\mathrm{MgO}$ layers leads to complex finite-size effects and temperature-dependent magnetic properties which must be carefully controlled for practical applications. In this article, we investigate the electronic, structural, and magnetic properties of $\mathrm{MgO} / \mathrm{Fe} / \mathrm{MgO}$ sandwiches using first-principles calculations and atomistic spin modeling based on a fully parametrized spin Hamiltonian. We find a large contribution to the effective interfacial magnetic anisotropy from the two-ion exchange energy. Minimization of the total energy using atomistic simulations shows a surprising spin-spiral ground-state structure at the interface owing to frustrated ferromagnetic and antiferromagnetic interactions, leading to a reduced Curie temperature and strong layerwise temperature dependence of the magnetization. The different temperature dependences of the interface and bulklike layers results in an unexpected nonmonotonic temperature variation of the effective magnetic-anisotropy energy and temperature-induced spin-reorientation transition to an in-plane magnetization at low temperatures. Our results demonstrate the intrinsic physical complexity of the pure $\mathrm{Fe} / \mathrm{MgO}$ interface and the role of elevated temperatures providing insight when interpreting experimental data of nanoscale magnetic tunnel junctions.

DOI: 10.1103/PhysRevApplied.9.054048

\section{INTRODUCTION}

The control of perpendicular magnetocrystalline anisotropy (PMCA) at ferromagnetic transition-metal-insulator interfaces is of paramount importance in the manufacture of spintronic devices, such as perpendicular magnetic tunnel junctions [1-3] and tunneling anisotropic magnetoresistive systems [4]. Large PMCA can be achieved by fabricating heterostructures including heavy nonmagnetic elements with large spin-orbit coupling (SOC) [5], such as $\mathrm{Co} / \mathrm{Pt}$ or $\mathrm{Co} / \mathrm{Au}$ [6]. It has been shown that this out-of-plane

*ramon.cuadrado@icn2.cat
PMCA enhancement at the interface is due to an increase of orbital moment of Co atoms and strong hybridization of the $3 d-5 d$ orbitals between the transition metals and the heavy atoms [6-9]. Recently, it has been demonstrated that other $3 d$ transition metal elements show increased PMCA even if their spin-orbit coupling is weak [10-12]. Such is the case of Fe-based thin films at $\mathrm{MgO}(001)$ interfaces, where the $\mathrm{Fe} d_{z^{2}}-\mathrm{O} p_{z}$ hybridization at the interfaces results in enhanced PMCA [13].

Using $a b$ initio calculations Yavorsky and Mertig [14] have shown the existence of antiferromagnetic $\mathrm{Fe} / \mathrm{Fe}$ interactions in the Fe plane connected to the $\mathrm{MgO}$. From this, they inferred the intriguing possibility of noncollinear 
magnetic structures but did not determine the ground-state spin structure. In addition to the possibility of antiferromagnetic interactions, the ground-state spin configuration will be perturbed due to the complex distance-dependent exchange interactions, which is difficult to quantify in layered systems with broken out-of-plane translational invariance. Spin fluctuations at elevated temperatures, coupled with the effects of any noncollinearity, will naturally lead to complex temperature-dependent behavior of the magnetic properties, including the magnetocrystalline anisotropy energy (MAE).

While there are a large number of applications of magnetic-nonmagnetic interfaces such as generating Skyrmions [15] or as magnetic tunnel junctions [16] in magnetic random-access memories, the physical properties of such interface systems (including $\mathrm{Fe} / \mathrm{MgO}$ ) are not fully understood. At the electronic level there exists a basic understanding of the interface exchange [14] and anisotropy [17], with very little understanding of thermal and long-range magnetic-ordering effects. Detailed understanding of the magnetization dynamics and temperature-dependent magnetic properties, particularly that of the magnetization and anisotropy, require a multiscale approach based on atomistic spin dynamics [18] with $a b$ initio parametrization. This is especially important given the localization of the magnetic anisotropy at the interface and the possibility of noncollinear spin structures.

In this article, we use a multiscale approach to model the electronic, structural, and magnetic properties of the $\mathrm{Fe} / \mathrm{MgO}$ interface in a sandwich geometry. Our results reveal the role of single-ion and two-ion anisotropy contributions, as well as the long-range exchange interactions in the ground-state configurations of two different bcc-Fe thicknesses sandwiched by two $\mathrm{MgO}(001)$ regions: $\cdots \mathrm{MgO} / n_{\mathrm{Fe}} \mathrm{Fe} / \mathrm{MgO} \cdots\left(n_{\mathrm{Fe}}=4,8\right)$. We show that the lack of Fe out-of-plane symmetry and dissimilar in-plane lattice constants compared to the Fe bulk have a drastic effect on the magnetic properties at the $\mathrm{Fe} / \mathrm{MgO}$ interface, leading to an exchange anisotropy which provides a dominant contribution to the total PMCA. Through atomistic simulations we show that the complex interfacial exchange interactions lead to a spiral ground-state spin structure. The temperature dependence of the anisotropy is shown to be nonmonotonic, driven by the different temperature dependences of the single-ion and two-ion anisotropies and leads to a significant reduction in the Curie temperature of the system as observed experimentally.

\section{DETAILS OF THE CALCULATIONS}

We use the SIESTA software package [19] to obtain the final $\cdots \mathrm{MgO} / n_{\mathrm{Fe}} \mathrm{Fe} / \mathrm{MgO} \cdots$ configurations used in the screened Korringa-Kohn-Rostoker (SKKR) calculations [20] by performing fully ionic conjugate gradient relaxation (see Sec. I of the Supplemental Material [21]). For the calculations we use a high number of $\mathrm{MgO}$ planes since the
SKKR method requires the system to be considered as an intermediate layer region positioned between two semiinfinite bulk regions. From the fully relaxed geometry, a $9 \mathrm{MgO} / n_{\mathrm{Fe}} \mathrm{Fe} / 9 \mathrm{MgO}$ slice is extracted and embedded into the $\mathrm{MgO}$ bulk, resulting in an Fe layer sandwiched between two semi-infinite $\mathrm{MgO}$ bulk alloys. The presence of the $\mathrm{MgO}$ interface leads to enhanced interfacial magnetic and orbital moments, giving total moments of approximately $2.8 \mu_{B}$ at the interface compared to approximately $2.3 \mu_{B}$ in the middle of the sandwich, as detailed in the Supplemental Material.

The layer-resolved exchange [22] and anisotropy contributions we calculate using the SKKR code (see Sec. I of the Supplemental Material for details [21]), allowing for a mapping onto a classical spin Hamiltonian [23,24]

$$
\mathcal{H}=-\sum_{i<j} \mathbf{S}_{i} \mathcal{J}_{i j} \mathbf{S}_{j}-\sum_{i} k_{i}\left(\mathbf{S}_{i} \cdot \mathbf{e}\right)^{2},
$$

where $\mathbf{S}_{i}$ is a classical unit vector parallel to the magnetization at site $i, \mathcal{J}_{i j}$ is the exchange-interaction tensor between sites $i$ and $j$, and the last term represents the onsite anisotropy, with the anisotropy constant $k_{i}$ and $\mathbf{e}$ the easy axis direction. Within the SKKR formalism, the total MAE, defined as the total energy difference between hard and easy magnetization directions, can be resolved into layerwise contributions $K_{l}$

$$
K=\sum_{l} K_{l}=K_{\text {on site }}+K_{\text {two site }},
$$

where $K_{\text {on site }}=\sum_{i} k_{i}$ and $K_{\mathrm{two} \mathrm{site}}$ is given an exchange anisotropy by the difference in the $z z$ and $x x$ components of the exchange tensor.

$$
K_{\mathrm{two} \mathrm{site}}=-\sum_{i<j}\left(J_{i j}^{x x}-J_{i j}^{z z}\right)
$$

\section{RESULTS}

\section{A. Layered resolved magnetic anisotropy}

The layer and site-resolved anisotropy contributions are shown in Fig. 1 for (a) $n_{\mathrm{Fe}}=4$ and (b) $n_{\mathrm{Fe}}=8$ layers. The $\mathrm{Mg}$ and $\mathrm{O}$ atoms make a negligible contribution to the MAE. At the interface, specifically on the first two Fe planes, the on-site values (blue filled squares) present higher values compared to those in the center for any configuration. In addition, these positive values imply that the Fe atoms contribute to the total MAE with an out-ofplane anisotropy. However, as the thickness increases, the on-site anisotropy in the center becomes more complex and for $n_{\mathrm{Fe}}=8$ the easy axis lies in-plane.

For four Fe planes $\left(n_{\mathrm{Fe}}=4\right)$, the on-site contribution to the MAE is four times larger than that of the $n_{\mathrm{Fe}}=8$ case, which we attribute to the enhanced orbital magnetic moment from the small number of Fe planes in the system. 

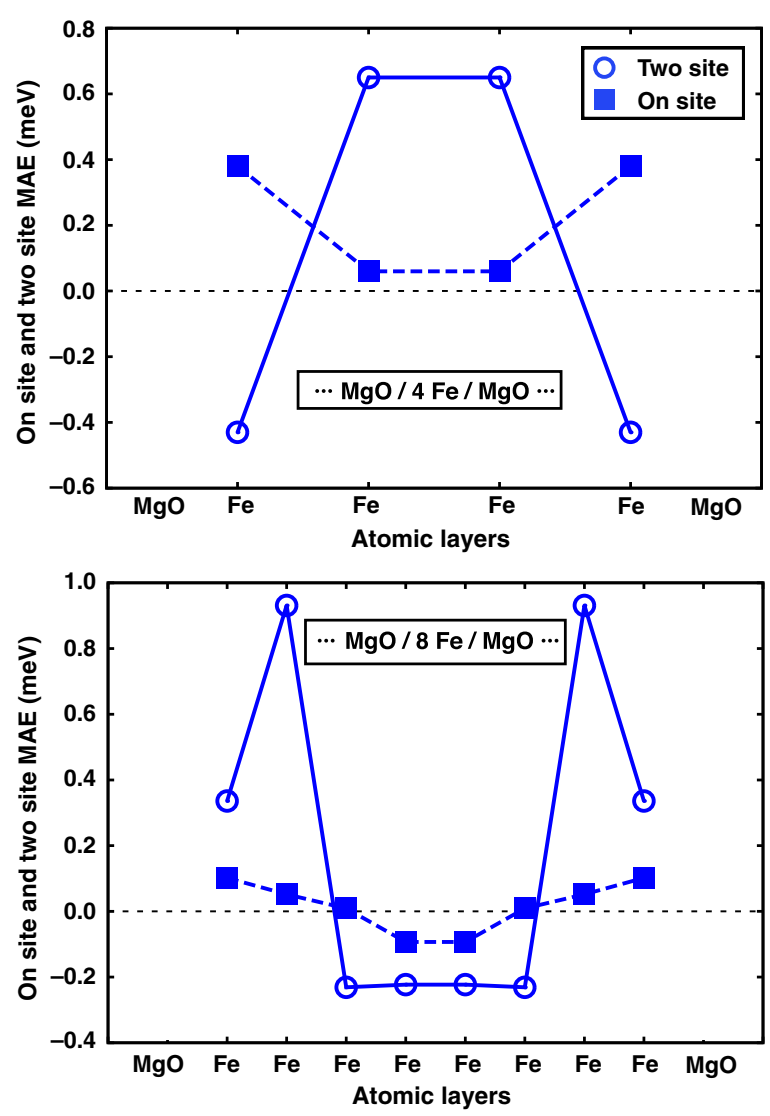

FIG. 1. On-site and two-site MAE contributions for $\cdots \mathrm{MgO} / n_{\mathrm{Fe}} \mathrm{Fe} / \mathrm{MgO} \cdots\left(n_{\mathrm{Fe}}=4,8\right)$.

The thickness dependence of the two-site anisotropy is more complicated, showing a sign change for the interface layer between $n_{\mathrm{Fe}}=4$ and $n_{\mathrm{Fe}}=8$ layers and also the appearance of a preferred in-plane orientation of the magnetization at the center of the $n_{\mathrm{Fe}}=8$ layer sandwich. In all cases the two-ion contributions to the MAE are seen to be dominant. Because of the different temperaturedependent scalings of single-ion and two-ion anisotropies [25] with the magnetization $\left(\mathrm{m}^{3}\right.$ and $\mathrm{m}^{2}$ at low temperature, respectively) their relative magnitudes determine the overall temperature dependence of the PMCA. Most ab initio calculations do not distinguish the origin of the PMCA, only its magnitude and, therefore, simple assumptions for the macroscopic temperature dependence of the anisotropy based on Callen-Callen theory [26] are likely to be erroneous. It is worth mentioning that other theoretical works have been performed in order to gain more insight into the nature of PMCA at Fe/MgO interfaces [17] and at $\mathrm{CoFe} / \mathrm{MgO}$ interfaces for different compositions [27]. In good agreement with our results (see Fig. 1), both works conclude that the main contributions to the anisotropy energy come from the two ferromagnetic layers closest to the interface. Zhang et al. [27] found that the PMCA at $\mathrm{Co}_{1_{x}} \mathrm{Fe}_{x} / \mathrm{MgO}$ interfaces strongly depends on the composition of $\mathrm{CoFe}$, decreasing with increasing $\mathrm{Co}$

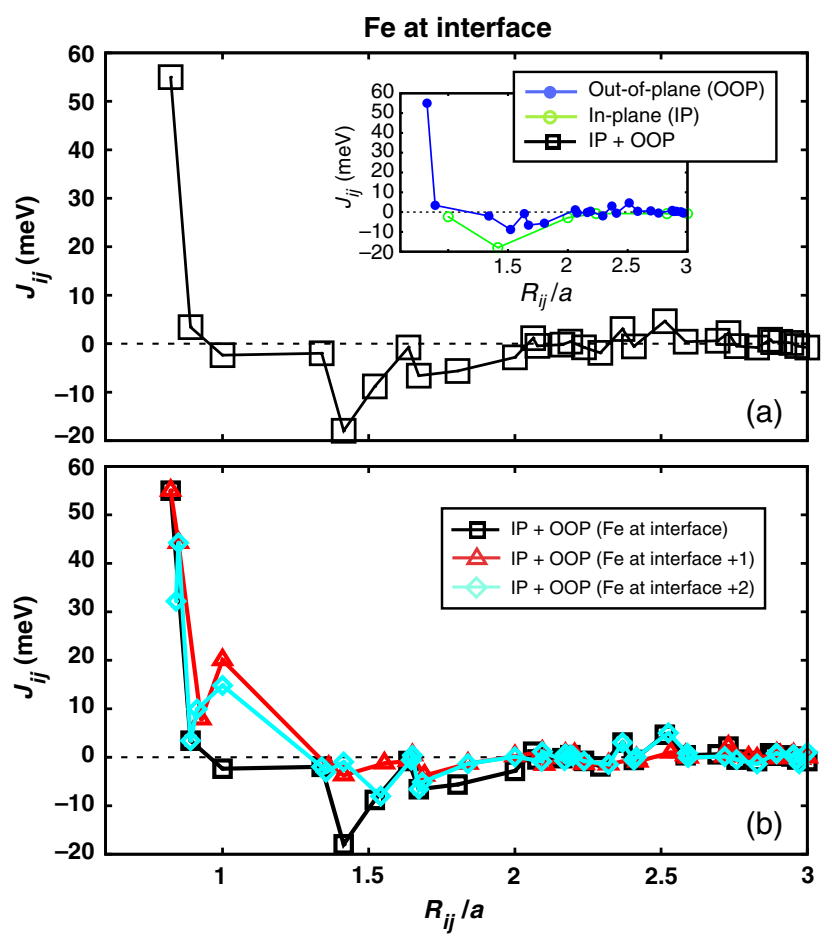

FIG. 2. (a) Fe-Fe isotropic magnetic exchange interactions as a function of the neighbor distances for an $\mathrm{Fe}$ atom located at the interface of the $\cdots \mathrm{MgO} / 8 \mathrm{Fe} / \mathrm{MgO} \cdots$ configuration. The inset shows the intralayer and interlayer isotropic exchange contributions. (b) Fe-Fe isotropic magnetic exchange interactions for $\mathrm{Fe}$ at interface (black squares), at interface +1 (red triangles), and at interface +2 (turquoise diamonds). The solid lines are guides for the eye.

concentration. Moreover, at a certain $x$ value there is a transition from positive to negative MCA. Hallal and co-workers [17] found that, besides the main interfacial contribution, the anisotropy energy propagates into the ferromagnetic bulk, showing an attenuating oscillatory behavior. In the present work (see the entry for $n_{\mathrm{Fe}}=8$ in Fig. 1) we also clearly observe this oscillatory behavior of the anisotropy energy.

\section{B. Magnetic exchange interactions}

In addition to site-resolved anisotropies, we study the effect of the $\mathrm{MgO} / \mathrm{Fe}$ interface on the interatomic exchange interactions. To aid analysis, we consider the trace of the exchange tensor $J_{i j}^{\text {iso }}=\frac{1}{3} \operatorname{Tr} \mathcal{J}_{i j}$ which forms the dominant part of the exchange energy. The range dependence of the exchange energy for the $n_{\mathrm{Fe}}=8$ system for different layers is shown in Fig. 2(a). As is commonly observed, the exchange interactions are long ranged and oscillatory in nature but also dominated by interactions with the nearest magnetic moments and decay rapidly with interatomic distance. The leading terms for all layers are ferromagnetic, leading to a general expectation of ferromagnetic ordering as would be expected for Fe. For bulk Fe, the exchange, as 
also shown by Yavorsky et al. [14]), shows antiferromagnetic contributions with the behavior becoming oscillatory after a couple of lattice spacings. However, in the $\mathrm{Fe} / \mathrm{MgO}$ interface system, the exchange remains antiferromagnetic for interactions out to a much longer range. To better resolve the origin of the ferromagnetic interactions we have separated the exchange interactions by layer in Fig. 2(b). This separation clearly shows that the large antiferromagnetic interactions in the interface layer occurs between atoms in the same plane. For the smaller $n_{\mathrm{Fe}}=4$ system, the nearest-neighbor interactions are similar, while the inplane antiferromagnetic interactions are $40 \mathrm{meV}$ larger, likely leading to a qualitatively different magnetic structure. In general, the isotropic $\mathrm{Fe}$ exchange at the interfaces for any $n_{\mathrm{Fe}}$ behaves in the same way, i.e., from the third Fe layer the magnetic behavior is similar to any Fe atom within the bulk and the main differences in the magnetic properties arise at the interface.

\section{Mesoscopic temperature dependence}

While the $a b$ initio calculations give information about the nature of the interactions in the system, their complex nature makes it difficult to draw clear conclusions about the macroscopic magnetic properties of the system as the ground state is governed by the balance of the whole set of interactions. The ab initio calculated Hamiltonian can then be used to determine the ground-state structure at elevated temperatures through atomistic spin modeling. The calculations utilize the VAMPIRE software package [28,29] considering a $10 \mathrm{~nm} \times 10 \mathrm{~nm} \times 8$ monolayer (ML) system of Heisenberg spins with periodic boundary conditions in the plane. We consider the full exchange tensor of over 2000 interactions per spin, localized anisotropy, and magnetic moments in the simulations. Metropolis and constrained [25] variants of Monte Carlo algorithms are used to determine the ground-state spin configurations in a plane-by-plane manner as well as the Curie temperature and temperature-dependent anisotropy.

A visualization of the final magnetic ground states for $n_{\mathrm{Fe}}=8$ are shown in Figs. 3(a) and 3(b). In (a) the top plane represents $\mathrm{Fe}$ at the $\mathrm{MgO}$ interface where a spinspiral ground state appears due to the frustration between ferromagnetic and antiferromagnetic interactions and the appearance of Dzyaloshinkii-Moriya interactions (DMI) $[30,31]$ at the interface due to broken inversion symmetry. The temperature dependence of the spin-spiral state is discussed in Sec. IV of the Supplemental Material [21]. Interestingly, the orientation of the spiral is at approximately $16.4^{\circ}$ to the $x$ axis and the spin spiral is confined to the positive $z$ values $\left(S_{z}>0\right)$. Moving layerwise towards the center of the sandwich, the spin spiral becomes much less prominent and a ferromagnetic ordering dominates, which confirms that the interfacial antiferromagnetic nearest-neighbor interactions and DMI are responsible for the frustrated spin-spiral structure. In fact, such a complex (a)

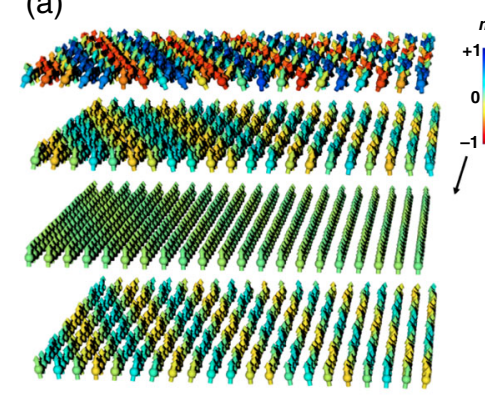

(b)

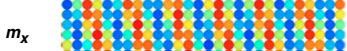

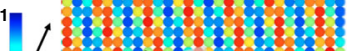
$\{t=1.2 n m$ ? ?

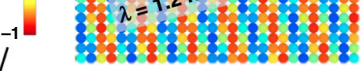

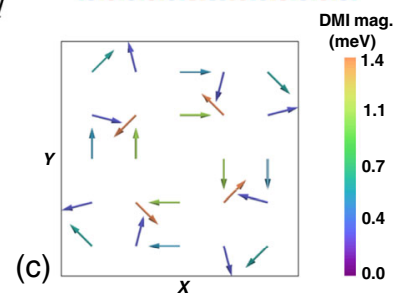

FIG. 3. Perspective (a) and top (b) view of the simulated ground-state spin structure at the $n_{\mathrm{Fe}}-\mathrm{Fe} / \mathrm{MgO}$ interface for $n_{\mathrm{Fe}}=8$, leading to a near-ferromagnetic state in the center of the sandwich. The coloring indicates the $x$ component of the magnetization (blue for $+x$, red for $-x$, and green for $x=0$ ). The atomic moments form a noncollinear configuration with wavelength $\lambda=1.2 \mathrm{~nm}$ and angle between spins no larger than $45^{\circ}$ in the perpendicular direction. (c) Plot of Dzyaloshinsky-Moriya interactions (DMI), which lay in the $x-y$ plane solely, for the top layer of the same system.

exchange pattern arises only in those layers that are at the interface with $\mathrm{MgO}$. Figure 3(c) shows the top view of the DMI magnitude for the top layer, where a nontrivial relationship among the neighbors of both direction and magnitude of DMI can be seen, and, as they lie in the $x-y$ plane, they can induce noncollinear spin configurations.

Using the spin model, we also investigate the effects of the anisotropy contributions on the overall effective value (see Fig. 4). The anisotropy is calculated using the constrained Monte Carlo method [25], an approach that allows

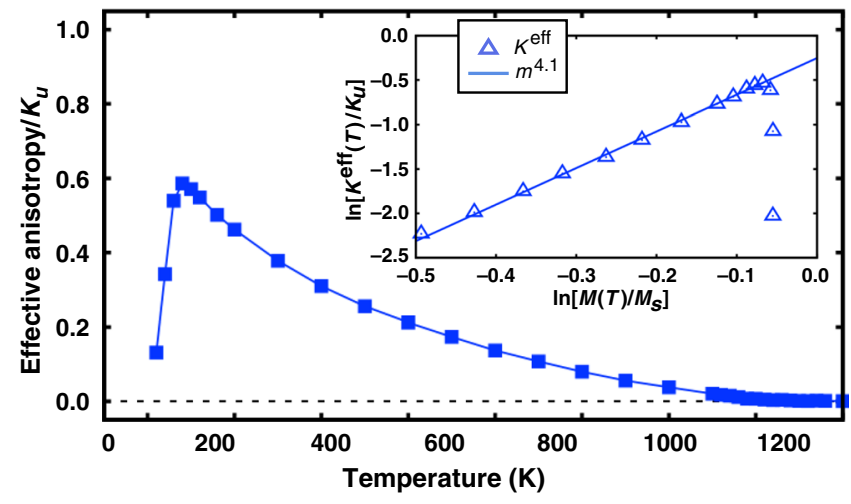

FIG. 4. Temperature dependence of the effective anisotropy as function of temperature for the $n_{\mathrm{Fe}}=8$ system; lines provide a guide for the eye. The inset shows the scaling of the anisotropy with the total magnetization; the symbols represent the data; and the solid line the fit. The simulations are performed by first equilibrating the system for 10000 Monte Carlo steps at each temperature and angle, and then the average of the torque is calculated over 50000 steps. 
one to constrain the direction of the magnetization away from the easy axis of the system. The anisotropy shows a surprising nonmonotonic behavior at low temperatures caused by the spin-spiral state. As the temperature-driven spin fluctuations increase, this destabilizes the spin-spiral ground state leading to an increase in the effective magnetic anisotropy as the magnetic ordering in the interface layer becomes more ferromagneticlike. Interestingly, the scaling of the effective magnetic anisotropy has an unusual exponent of $k(m) m^{4.1}$, which is expected for neither single-ion $\left(\mathrm{m}^{3}\right)$ or two-ion $\left(\mathrm{m}^{2}\right)$ anisotropy scaling. We attribute this unusual exponent to the frustrated nature of the exchange interactions at the interface, and so we expect a high exponent for the interface layer and lower for the subsurface layers, which when normalized to the average magnetization gives a higher effective scaling of the anisotropy constant.

\section{CONCLUSION}

In conclusion, we present a fully relativistic electronic structure study of the exchange interactions and the siteresolved MAE of $\cdots \mathrm{MgO} / n_{\mathrm{Fe}} \mathrm{Fe} / \mathrm{MgO} \cdots\left[n_{\mathrm{Fe}}=4,8\right]$ sandwiches. Our results reveal a dominant contribution to the MAE from the two-ion exchange anisotropy. Furthermore, we show that the competing ferromagnetic and antiferromagnetic interactions at the $\mathrm{Fe} / \mathrm{MgO}$ interface lead to an overall ferromagnetic ground state but which includes a frustrated spin spiral at the interface, which destabilizes the magnetization of the interfacial layer at elevated temperatures, leading to a reduction of the Curie temperature of the system. The complex equilibrium magnetic properties of this technologically important material are of critical importance to understanding the thermal stability and spin-transport properties of nanoscale MTJ devices and can provide key insights into experimental observations.

\section{ACKNOWLEDGMENTS}

The financial support of the Samsung Global MRAM Innovation programme and the European Union under the Seventh Framework Programme under Grant No. 281043, FEMTOSPIN is gratefully acknowledged. ICN2 is supported by the Severo Ochoa program from Spanish MINECO (Grant No. SEV-2013-0295). R. C. also acknowledges the funding from the European Unions Horizon 2020 research and innovation program under the Marie Skłodowska-Curie Grant Agreement No. 665919. T. A. Ostler gratefully acknowledges the Marie Curie incoming BeIPD-COFUND fellowship program at the University of Liège. A. D., L. O., and L. S. acknowledge support by the Hungarian Scientific Research Fund (NKFIH) under Projects No. K115575, No. PD124380, No. K108676, No. K115608, and No. FK124723. L. O. also acknowledges to the National Quantum Technologies Program
NKP-375 2017-00001 of the NRDI Office of Hungary. L. O. acknowledges support from the Janos Bolyai Scholarship of the Hungarian Academy of Sciences. L. S. and A. D. are grateful for financial support by the BME-Nanonotechnology FIKP grant of EMMI (BME FIKP-NAT).

[1] K. Mizunuma, S. Ikeda, J. H. Park, H. Yamamoto, H. Gan, K. Miura, H. Hasegawa, J. Hayakawa, F. Matsukura, and H. Ohno, $\mathrm{MgO}$ barrier-perpendicular magnetic tunnel junctions with $\mathrm{CoFe} / \mathrm{Pd}$ multilayers and ferromagnetic insertion layers, Appl. Phys. Lett. 95, 232516 (2009).

[2] J.-H. Park, C. Park, T. Jeong, M. T. Moneck, N. T. Nufer, and J.-G. Zhu, CoPt multilayer based magnetic tunnel junctions using perpendicular magnetic anisotropy, J. Appl. Phys. 103, 07A917 (2008).

[3] G. Kim, Y. Sakuraba, M. Oogane, Y. Ando, and T. Miyazaki, Tunneling magnetoresistance of magnetic tunnel junctions using perpendicular magnetization $\mathrm{L}_{0}-\mathrm{CoPt}$ electrodes, Appl. Phys. Lett. 92, 172502 (2008).

[4] L. Gao, X. Jiang, S.-H. Yang, J. D. Burton, E. Y. Tsymbal, and Stuart S.P. Parkin, Bias Voltage Dependence of Tunneling Anisotropic Magnetoresistance in Magnetic Tunnel Junctions with $\mathrm{MgO}$ and $\mathrm{Al}_{2} \mathrm{O}_{3}$ Tunnel Barriers, Phys. Rev. Lett. 99, 226602 (2007).

[5] J. Stöhr and H. C. Siegmann, Magnetism (Springer, Berlin, 2006).

[6] I. Zutic, J. Fabian, and S. Das Sarma, Spintronics: Fundamentals and applications, Rev. Mod. Phys. 76, 323 (2004).

[7] D. Weller, Y. Wu, J. Stohr, M. G. Samant, B. D. Hermsmeier, and C. Chappert, Orbital magnetic moments of $\mathrm{Co}$ in multilayers with perpendicular magnetic anisotropy, Phys. Rev. B 49, 12888 (1994).

[8] C. J. Aas, P. J. Hasnip, R. Cuadrado, E. M. Plotnikova, L. Szunyogh, L. Udvardi, and R. W. Chantrell, Exchange coupling and magnetic anisotropy at $\mathrm{Fe} / \mathrm{FePt}$ interfaces, Phys. Rev. B 88, 174409 (2013).

[9] R. Cuadrado and R. W. Chantrell, Interface magnetic moments enhancement of FePt-L10/MgO(001): An ab initio study, Phys. Rev. B 89, 094407 (2014).

[10] K. Nakamura, T. Akiyama, T. Ito, M. Weinert, and A. J. Freeman, Role of an interfacial FeO layer in the electricfield-driven switching of magnetocrystalline anisotropy at the Fe/MgO interface, Phys. Rev. B 81, 220409(R) (2010).

[11] B. Rodmacq, S. Auffret, B. Dieny, S. Monso, and P. Boyer, Crossovers from in-plane to perpendicular anisotropy in magnetic tunnel junctions as a function of the barrier degree of oxidation, J. Appl. Phys. 93, 7513 (2003).

[12] M. K. Niranjan, C.-G. Duan, S. S. Jaswal, and E. Y. Tsymbal, Electric field effect on magnetization at the $\mathrm{Fe} /$ MgO(001) interface, Appl. Phys. Lett. 96, 222504 (2010).

[13] Kohji Nakamura, Yushi Ikeura, Toru Akiyama, and Tonomori Ito, Giant perpendicular magnetocrystalline anisotropy of $3 \mathrm{~d}$ transition-metal thin films on $\mathrm{MgO}$, J. Appl. Phys. 117, 17C731 (2015).

[14] B. Yu. Yavorsky and I. Mertig, Noncollinear interface magnetism and ballistic transport in $\mathrm{FeFeOMgOFe}$ tunnel 
junctions: Ab initio calculations using the KKR method, Phys. Rev. B 74, 174402 (2006).

[15] B. Dupé, G. Bihlmayer, M. Böttcher, S. Blügel, and S. Heinze, Engineering Skyrmions in transition-metal multilayers for spintronics, Nat. Commun. 7, 11779 (2016).

[16] S. Ikeda, K. Miura, H. Yamamoto, K. Mizunuma, H. D. Gan, M. Endo, S. Kanai, J. Hayakawa, F. Matsukura, and H. Ohno, A perpendicular-anisotropy CoFeB-MgO magnetic tunnel junction, Nat. Mater. 9, 721 (2010).

[17] A. Hallal, H. X. Yang, B. Dieny, and M. Chshiev, Anatomy of perpendicular magnetic anisotropy in $\mathrm{Fe} / \mathrm{MgO}$ magnetic tunnel junctions: First-principles insight, Phys. Rev. B 88, 184423 (2013).

[18] N. Kazantseva, D. Hinzke, U. Nowak, R. W. Chantrell, U. Atxitia, and O. Chubykalo-Fesenko, Towards multiscale modeling of magnetic materials: Simulations of FePt, Phys. Rev. B 77, 184428 (2008).

[19] J. M. Soler, E. Artacho, J. D. Gale, A. García, J. Junquera, P. Ordejón, and D. Sánchez-Portal, The SIESTA method for ab initio order- $N$ materials simulation, J. Phys. Condens. Matter 14, 2745 (2002).

[20] J. Zabloudi, R. Hammerling, L. Szunyogh, and P. Weinberger, Electron Scattering in Solid Matter (Springer, Berlin, 2005).

[21] See the Supplemental Material at http://link.aps.org/ supplemental/10.1103/PhysRevApplied.9.054048 for details of the calculation procedures.

[22] L. Udvardi, L. Szunyogh, K. Palotás, and P. Weinberger, First-principles relativistic study of spin waves in thin magnetic films, Phys. Rev. B 68, 104436 (2003).
[23] A. I. Liechtenstein, M. I. Katnelson, V. P. Antropov, and V. A. Gubanov, Local spin density functional approach to the theory of exchange interactions in ferromagnetic metals and alloys, J. Magn. Magn. Mater. 67, 65 (1987).

[24] J. B. Staunton, L. Szunyogh, A. Buruzs, B. L. Gyorffy, S. Ostanin, and L. Udvardi, Temperature dependence of magnetic anisotropy: An ab initio approach, Phys. Rev. B 74, 144411 (2006).

[25] P. Asselin, R. F. L. Evans, J. Barker, R. W. Chantrell, R. Yanes, O. Chubykalo-Fesenko, D. Hinzke, and U. Nowak, Constrained Monte Carlo method and calculation of the temperature dependence of magnetic anisotropy, Phys. Rev. B 82, 054415 (2010).

[26] H. B. Callen and E. Callen, The present status of the temperature dependence of magnetocrystalline anisotropy, and the power law, J. Phys. Chem. Solids 27, 1271 (1966).

[27] Jia Zhang, Christian Franz, Michael Czerner, and Christian Heiliger, Perpendicular magnetic anisotropy in $\mathrm{CoFe} / \mathrm{MgO} /$ $\mathrm{CoFe}$ magnetic tunnel junctions by first-principles calculations, Phys. Rev. B 90, 184409 (2014).

[28] R. F. L. Evans, W. J. Fan, P. Chureemart, T. A. Ostler, M. O. A. Ellis, and R. W. Chantrell, Atomistic spin model simulations of magnetic nanomaterials, J. Phys. Condens. Matter 26, 103202 (2014).

[29] http://vampire.york.ac.uk.

[30] I. Dzyaloshinsky, A thermodynamic theory of weak ferromagnetism of antiferromagnetics, J. Phys. Chem. Solids 4, 241 (1958).

[31] T. Moriya, Anisotropic superexchange interaction and weak ferromagnetism, Phys. Rev. 120, 91 (1960). 